\title{
Anti-Hepatitis E Virus Markers in Hemodialysis Patients
}

\begin{tabular}{|l|l|l|}
\hline C.-H. & Chwan-Heng & Wang $^{\mathrm{a}}$ \\
\hline S.-Y. & Shu-Yuan & Tschen $^{\mathrm{b}}$ \\
\hline G. & Gunnar & Schalasta $^{\mathrm{c}}$ \\
\hline J. & Jacques & Pillot $^{\mathrm{d}}$ \\
\hline G. & Gerhart & Jahn $^{\mathrm{a}}$ \\
\hline B. & Bertram & Flehmig $^{\mathrm{a}}$ \\
\hline
\end{tabular}

${ }^{a}$ Abteilung für Medizinische Virologie und Epidemiologie der Viruskrankheiten, Hygiene Institut, Klinikum der Eberhard-Karls-Universität Tübingen, ${ }^{\mathrm{b}}$ Institut für Mikrobiologie I, Eberhard-Karls-Universität Tübingen, and 'Institut fur Virologie, Infektiologie und Epidemiologie, Stuttgart, Germany; 'Unité Immunologie Microbienne, WHO Center for Research and Reference on Viral Hepatitis, Institut Pasteur, Paris, France

Dr. Chwan-Heng Wang, Abteilung für Medizinische Virologie, Hygiene Institut, Klinikum der Eberhard-Karls-Universität Tübingen, Silcherstr. 7, D-72076 Tübingen (Germany)

Dear Sir,

The hepatitis E virus (HEV) causes enter-ically transmitted non-A, non-B hepatitis and has been identified in the excreta of acute hepatitis patients throughout the world, predominantly in tropical and subtropical countries. Several strains of HEV have been characterized according to their physi-cochemical and biological properties in such a way as to prove that HEV is independent of all known human hepatotropic viral agents, thus forming a separate genus tentatively designated as Hepevirus, a member of a completely new genus within the Caliciviridae family $[1,2]$.

The infection occurs epidemically and sporadically, usually through fecal contamination of water supplies. Although this hepatitis follows a benign and unremarkable course, it is also known to cause severe and fulminant hepatitis, which is often fatal, especially in pregnant women [2]. The unexpectedly high mortality rate observed in HEV-infected pregnant women may reflect a unique feature of viral replication or disease pathogenesis in these individuals. To determine this, further studies are needed to identify additional factors involved in the epidemiology and pathogenesis of hepatitis E.

This agent has not yet been implicated as a cause of hepatitis in western Europe, probably because of its standards of sanitation. However, it is not yet known whether the epidemiology of HEV infection is beginning to change following the political unification of eastern and western Europe. Whether such social changes and movability of human populations have had an impact on emerging infection rates remains to be established. Nevertheless, it is worth mentioning that preliminary seroprevalence studies with enzyme-conjugated immunoassays (enzyme immunoassay for anti-HEV) conducted worldwide suggest that hepatitis $\mathrm{E}$ is not confined to underdeveloped countries. Anti-HEV has been found in up to $10 \%$ of selected populations surveyed in regions of the world not previously known to harbor this virus and fulminant hepatic failure due to HEV infection has been documented in both British and 
American subjects who had not travelled to any known endemic regions [2, 3]. Although, at present, hepatitis $\mathrm{E}$ is not a major public health problem in industrialized countries, the increasing number of travellers to HEV-endemic areas and the high influx of immigrants from such countries necessitate a careful surveillance.

Several cases of HEV infection occurring in Europe during the past few years have been assumed to have been imported from the endemic, developing countries to these developed countries $[4,5]$. Also, indirect data concerning different cases of anti-HEV patients who had travelled abroad have been

recorded. In addition, several authors have provided indications that this disease could possibly be endemic in Europe, albeit with a low circulation [5]. For example, most of the positive cases in Italy were not associated with travels to endemic areas. These data suggest a possible low circulation of HEV in Europe, especially in its southern regions. However, transmission of HEV in Europe is rare, mysterious and remains unexplained.

In one exceptional case, the transmission of HEV in a hospital has also been provisionally associated with health care devices and surgical procedures [4]. Recently, in our hospital, a female clinical worker was demonstrated to have a very high titer of anti-HEV, which lasted for at least 6 months. She had not travelled abroad but had worked in a department with highly infectious patients. Speculations arose as to whether the hospital environment might have been contaminated with the virus through unspecified ways, but the question of how the real transmission of the HEV occurred in the country remains to be solved. Therefore, there are reasons for reconsidering the possible transmission of hepatitis E in hospitals.

Hepatitis was a serious problem in chronic dialysis units before the introduction of serological tests, vaccine for HBV infection, and universal precaution standards. In the past several months, we de-

\section{KAIUiEH}

E-Mail karger@karger.ch Fax+416130612 34

(c) 1996 S.KargerAG, Basel 0028-2766/96/0722-0343\$8.00/0

Fig. 1. Detection of HEV-RNA by reverse transcription and poly-merase chain reaction (PCR). 5 serum samples derived from five chronic dialysis patients containing positive anti-HEVs were tested. The putative capsid gene of HEV was amplified and PCR products were stained with ethidium-bromide on a 3\% agarose gel [9]. Lanes 1, 2 = Positive controls derived from a stool sample (2-round PCR); lanes 3, 4, and 12 = positive controls derived from HEV-RNA transcripts (2-round PCR); lane $5=1$ round of PCR of lane 1 ; lane $6=1$ round of PCR of lane 3; lanes 7-11 = serum samples from dialysis patients with positive anti-HEV (2-round PCR). Briefly, ribonucleic acid was extracted from each sample as follows: $100 \mu \mathrm{l}$ of each serum sample was dissolved in $400 \mu \mathrm{l}$ of lysis solution ( $5 \mathrm{~mol} / \mathrm{l}$ guanidium thiocyanate; $37.5 \mathrm{mmol} / \mathrm{l}$ sodium citrate, $\mathrm{pH} 7$; $0.75 \%$ sodium $\mathrm{N}$-lau-ryl sarcosine and $0.15 \mathrm{mmol} / \mathrm{l}$ 2-mercaptoethanol; $10 \mu \mathrm{g}$ of transfer RNA), followed by 2-propanol and ethanol precipitation. After washing with 70\% ethanol, the final RNA pellet was dissolved in $20 \mu \mathrm{l}$ of sterile deionized ribonuclease I-free water. Reverse transcription was performed on $5 \mu \mathrm{l}$ of each RNA sample for 30 min at $43{ }^{\circ} \mathrm{C}$ followed by 5 min at $95^{\circ} \mathrm{C}$. Initiation of transcription from the 3 ' end of the HEV genome was achieved with primer 5'-ACCCTATACCGGT-GCCCTC-3 (6019-6038), primer 5'GAGGTCAATGTCATGCG-G-3' (6425-6443) was added, and 35 cycles of PCR performed using Taq polymerase (Perkin-Elmer Cetus, Norwalk, USA) according to the manufacturer's 
instructions, with $10 \mathrm{~s}$ at $94^{\circ} \mathrm{C}, 10 \mathrm{~s}$ at $55^{\circ} \mathrm{C}$ and $20 \mathrm{~s}$ at $72^{\circ} \mathrm{C}$. For the second run, $10 \mu \mathrm{l}$ of each initial PCR reaction mix was diluted with $94 \mu \mathrm{l}$ of a new Taq polymerase reaction mix containing two internal primers 5'-AAGACCAATACGCG-GGTCT-3' (6085-6108) and 5'CAAGCAGAGTGTCAGCAAG-3' (6266-6285) [10]. The PCR was repeated with the conditions described above. Electrophoresis was performed on $10 \mu \mathrm{l}$ of each sample on a $3 \%$ agarose gel stained with ethidium bromide in order to identify the expected 423/196 base pair PCR product. A negative control (HEV-negative serum from a laboratory staff member) and one positive control of HEV-plasmid DNA were processed in parallel with the unknown samples. Alternatively, sense and anti-sense synthetic oligonucleotide primers corresponding to the nucleo-tide sequence of the putative HEV polymerase gene were used in reverse transcription PCRs for amplification of the viral genome. The primers used were antisense primer 5'TTCAACTTCAAGCCA-CAGCC-3' (1000-1019) and sense primer 5-GCGTGGATCTTGCAGGCC-3' (780-797) [10, 11]. In this study, HEV-RNA transcripts from a plasmid was used as positive control. HEV-RNA templates were constructed by incorporating at T7 promotor sequence into a PCR primer corresponding to the 3'-capsid region of the HEV genome [11].

tected 23 anti-HAV cases, using an anti-HEV immunoassay based on a recombinant HEV antigen [Diagnostic Biotechnology, Singapore, 6], from 400 serum samples submitted to the Tubingen Hospital. This result was further confirmed by an other assay based on the syntheticpeptide HEV antigen (South Pacific Institute for Biomedical Science). Etio-logically, these patients with positive anti-HEV in their blood had histories of hepatitis or subclinical histories. Surprisingly, among these patients, 10 cases were found in association with dialysis. It is not known whether the infections of these patients derived from dialysis treatment or whether they had a very high rate of blood transfusions. No patients in these groups were of foreign origin. Anti-HEV seems to have been obtained from the hemodialysis treatment, because a correlation exists between the time of treatment and

the anti-HEV. These serum samples were screened for anti-HEV because of its association with certain surgical procedures due to insufficient kidney function or nephrectomy. Two of these patients had been treated with dialysis for a very long time. These HEV infection cases seem to be very similar to cases of HCV transmission already observed in dialysis patients. Dialysis patients have been shown to have a very high risk of an HCV infection [7, 8]. In these studies, transfusion-associated HCV infection was found to represent $80 \%$ of the total hepatitis present. At that time, the remaining $20 \%$ of the hepatitis cases did not have any hepatitis markers (HAV, $\mathrm{HBV}$ or $\mathrm{HCV}$ ). When tests for known hepatitis viruses prove negative in many patients who have undergone dialysis, a non- $\mathrm{A}$, non- $\mathrm{B}$, non-C hepatitis virus may be responsible for provoking the hepatitis. In this report, we describe a significant prevalence of anti-HEV in some hemodialysis patients whose blood samples had been collected in the spring 1994.

To investigate HEV transmission, the successful identification of HEV in blood or sera at the scene of infection is absolutely necessary. Without this experimental confirmation, questions on whether HEV can be directly transmitted through blood in developed countries is still hypothetical. The prevalence among intravenous drug users (IVDUs) was demonstrated to be significantly higher than among blood donors and healthy people of a comparable age [5]. This higher prevalence among IVDUs might be due to poor hygiene but also possibly to a parenteral route of transmission. The sero-prevalence of antibodies to HEV is very high among IVDUs and individuals over 40 years 


\section{4}

Nephron 1996;72:343-345

Wang/Tschen/Schalasta/Pillot/Jahn/ Flehmig

of age. The fact of the high prevalence of anti-HEV among the drug users therefore strengthens the argument that contact with foreign blood can increase the transmission. This data seems to agree with our previous hypothesis, which was based on the preliminary observation that HEV might be able to be transmitted by blood.

$\mathrm{HEV}$ is an emerging virus taking advantage of unknown viral mechanisms for the evolution of its diversity. It has been proposed that atypical strains of HEV are appearing [9]. Through a lack of sequence data on regional isolates as in Europe or Africa, an accurate determination of the existence of the virus by using techniques such as poly-merase chain reaction is extremely difficult. However, in this study, we examined the HEV genome with primer pairs specific to the highly conservative regions of the putative polymerase gene and the capsid gene [10]. Here, polymerase chain reaction in both variations failed to detect HEV in the serum or stools of any of these patients (fig. 1) [11]. Considering the short viremic phase after infection, we are not suprised by an absence of HEV in the serum of these patients. Undetectable levels of anti-HEV IgM in these patients also provide an indication that the samples available for testing were taken after recovery of the disease and were not from the acute viremic phase.

The presence of HEV in serum even before clinical signs had appeared suggests that in endemic areas, the sporadic transmission of HEV may also occur parenterally. Unfortunately, our isolation of HEV in these patients has so far been unsuccessful. Due to the conceptual background regarding the in-

frequent occurrence of HEV infection in Europe and the limitations of serological tests, a 'lastminute' diagnosis is not always possible. Catching the viruses present in their viremic phase has proved difficult because of this diagnostic delay. However, many authors have shown without any doubt that HEV was found to be present in the blood [12]. And, in general, HEV can be transmitted by blood donated by infected individuals during the transient phase of viremia that precedes the onset of clinical symptoms. Accordingly, this data agrees with our previous views that in developed countries, regional transmission of the virus could occur through blood transfusions.

In this study, anti-HEV markers were found in some hemodialysis patients at the Tubingen Hospital. It is not known whether these patients contracted their infections from dialysis treatment or whether these patients had very high rates of blood transfusions or contact with contaminated devices or persons. Our results suggest that it is possible that HEV is transmitted by blood contamination. The transmission vehicles seem to be very similar to those responsible for the transmission of the HCV previously observed in dialysis patients.

Acknowledgements

This work was supported by a fortune program of our university hospital. Dr. S.-Y. Tschen was supported in part by a grant from the Chung-Die fund. Lastly, we would like to thank Dr. J.W. Chen for his gift of a synthetic peptide-based anti-HEV assay.

References

Balayan MS: Hepatitis E virus: Some characteristics relevant to virus classification. VIII Int Symp Viral Hepatitis Liver Dis (abstract B25), Tokyo, 1993.

Bradley D, Purdy M, McCaustland K, Krawc-zynski K: Hepatitis E virus: Recent advances in the epidemiology, biology, and molecular biology of the major causative agent of enterically 
transmitted non-A, non-B hepatitis. VIII Int Symp Viral Hepatitis Liver Dis (abstract P6), Tokyo, 1993.

Hibbs JR, Frickhofen N, Rosenfeld SJ, et al: Aplastic anemia and viral hepatitis: Non-A, non-B, non-C. JAMA 1992;267:2051-2054.

Robson SC, Adams S, Brink N, Woodruff B, Bradley D: Hospital outbreak of hepatitis E. Lancet 1992;339:1424-1425.

Zanetti AR, Dawson GJ: Hepatitis type E in Italy: Seroepidemiological survey. J Med Virol 1994;42:318-320.

Wang CH, Flehmig B, Moeckli R: Transmission of hepatitis E virus by transfusion. Lancet 1993;341:825-826.

Calabrese G, Vagelli G, Guaschino R, Gonella M: Transmission of anti-HCV within the household of haemodialysis patients. Lancet 1991;338:1466.

Huang CH, Ho MS, Yang CS, Lee CL, Tan CA: Hepatitis C markers in hemodialysis patients. J Clin Microbiol 1993;31:1764-1769.

Chauhan A, Dilawari JB, Kaur U, et al: Atypical strain of hepatitis E virus (HEV) from North India. J Med Virol 1994;44:22-29.

Tarn AW, Smith MM, Querra ME, et al: Hepatitis E virus (HEV): Molecular cloning and sequencing of the full-length viral genome. Virology 1991;185:120-131.

Wang CH, et al: Quantitation of HEV genome in a stool sample. In preparation.

Schlauder GG, Dawson GJ, Mushahwar IK, et al: Viraemia in Egyptian children with hepatitis E virus infection. Lancet 1993;341:378.

Anti-HEV Markers in Hemodialysis Patients

345 